\title{
EXPLICIT STIFFNESS MATRIX FOR PARABOLIC PRISMATIC TRIANGULAR ELEMENT
}

\section{Marcelo R. de Matos Pedreiro ${ }^{a}$, Rogério de O. Rodrigues ${ }^{b}$, Maicon Marino Albertini ${ }^{\mathrm{c}}$ e Jefferson S. Camacho ${ }^{\mathrm{b}}$}

${ }^{a}$ Universidade Camilo Castelo Branco, Campus VII, Estrada Projetada F1, s/n, Fazenda Santa Rita, 15600-000, Fernandópolis, SP, Brasil, marcelo.pedreiro@bol.com.br http://www.unicastelo.br

${ }^{b}$ Núcleo de Ensino e Pesquisa de Alvenaria Estrutural, Universidade Estadual Paulista "Júlio de Mesquita Filho", Avenida Brasil Centro, 56, Ilha Solteira, SP, Brasil, nepae@dec.feis.unesp http://www.feis.unesp.br

${ }^{\mathrm{C} U n i v e r s i d a d e}$ Camilo Castelo Branco, Campus VII, Estrada Projetada F1, s/n, Fazenda Santa Rita, 15600-000, Fernandópolis, SP, Brasil, maicon.albertini@unicastelo.br http://www.unicastelo.br

Keywords: Structural Mechanics, Finite Element Method, Volumetric Element.

Resumo. O presente trabalho tem como objetivo principal explicitar a matriz de rigidez para o elemento finito prismático triangular parabólico (Wedge-15 / W15), contendo quinze nós onde se consideram como graus de liberdade somente as translações em cada uma das três direções do espaço. Para facilitar a integração analítica, o elemento foi tratado com um mapeamento isoparamétrico com domínio em coordenadas naturais, cujas funções aproximadoras contêm quinze monômios extraídos do polinômio algébrico quadrático completo em $x$, y e z. Ao longo da dedução analítica se faz necessário mudar o domínio e os limites de integração através da transformação de coordenadas utilizando-se a matriz Jacobiana. Compete também a este trabalho explicitar as matrizes de forças nodais equivalentes para aplicação de carregamentos superficiais no elemento. A validação da matriz de rigidez explicita é feita através de exemplos onde sejam aplicáveis as equações provenientes da teoria de Resistência dos Materiais, sendo os resultados observados comparados com os obtidos utilizando-se modelos discretizados com o elemento desenvolvido, aplicando-se diferentes formas de discretização e carregamento. A obtenção de tal matriz permitirá a análise estática ou dinâmica de sistemas estruturais em geral, seja linear ou não-linear, de forma mais eficiente quando comparada com a utilização de matriz obtida numericamente. 


\section{INTRODUÇÃO}

O Método dos Elementos Finitos - MEF foi idealizado com os trabalhos de Argyris e Kelsey (1954, apud RODRIGUES 1997, p.1 [5]) e de Turner et al (1956, apud RODRIGUES 1997, p.1 [5]). Com isso os pesquisadores passaram a ter uma ferramenta poderosa que permite a modelagem numérica dos fenômenos envolvidos na análise estrutural. O MEF baseia-se na discretização do sistema estrutural, onde seus componentes são divididos em pequenas regiões, chamadas de elementos finitos, sendo essas interconectadas entre si por meio de nós para formar o conjunto estrutural, conforme visto em [6]. O processo de discretização é a base do conceito MEF garantindo uma melhor convergência dos resultados para a resposta real do modelo.

Nesse contexto, a utilização de elementos finitos volumétricos permite uma análise mais precisa do comportamento de estruturas diversas, como exemplo alvenaria estrutural conforme visto em [1], desprezado as hipóteses das teorias simplificadoras que são necessárias para uma discretização com elementos finitos lineares e laminares.

\section{ELEMENTO FINITO PRISMÁTICO TRIANGULAR PARABÓLICO}

O elemento finito prismático triangular parabólico de quinze nós, denominado Wedge 15 - W15, ilustrado na Figura 1, apresenta variação quadrática de deslocamentos ao longo de suas arestas, sendo que para cada nó são consideradas as três translações como graus de liberdade.

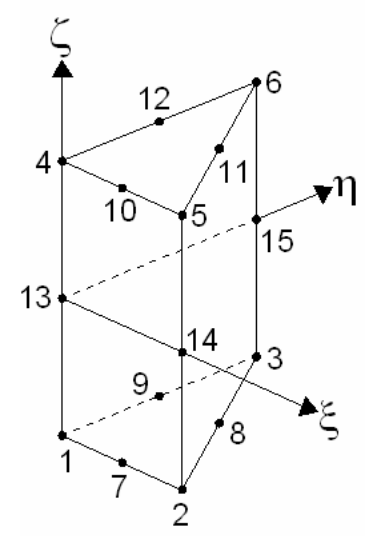

Figura 1 - Elemento finito W15 com quinze nós. Fonte: Pedreiro (2011)

A formulação do elemento é feita utilizando-se coordenadas homogêneas, o que torna a solução do problema mais simples, uma vez que os polinômios aproximadores ficam em função de coordenadas adimensionais. A definição das coordenadas homogêneas para o domínio triangular do elemento são apresentadas na integra no trabalho de PEDREIRO (2011) [4], aonde se chega à relação geral dada pela equação (1).

$$
\xi_{i}=\frac{1}{2 A}\left(a_{i}+m_{i} x+n_{i} y\right)
$$


As funções aproximadoras desse elemento contêm quinze monômios extraídos do polinômio algébrico cúbico completo em x, y e z. Nesse caso, para garantir a continuidade com os deslocamentos dos elementos adjacentes, a função deslocamento deve variar parabolicamente ao longo dos lados. Sendo assim, para um sistema de coordenadas adimensionais, com origem no centro do lado 1-4 do pentaedro, e usando-se a relação encontrada na equação (1), pode-se escrever com base na Figura 1 que:

$$
\xi=\xi_{2} \quad \text { e } \quad \eta=\xi_{3}
$$

e aplicando-se ao elemento em questão, pode-se escrever que:

$$
\xi=\frac{1}{2 A}\left(a_{2}+m_{2} x+n_{2} y\right) ; \eta=\frac{1}{2 A}\left(a_{3}+m_{3} x+n_{3} y\right) \text { e } \zeta=\frac{Z}{C}
$$

As funções interpoladoras para os deslocamentos u, v e w, são dadas pelas equações (4), (5) e (6), respectivamente, todos extraídos da pirâmide de Pascal, conforme visto em [2].

$$
\begin{aligned}
& u(\xi, \eta, \zeta)=\alpha_{0}+\alpha_{1} \xi+\alpha_{2} \eta+\alpha_{3} \zeta+\alpha_{4} \eta \zeta+\alpha_{5} \xi \zeta+\alpha_{6} \xi \eta+\alpha_{7} \xi^{2}+\alpha_{8} \eta^{2}+\alpha_{9} \zeta^{2} \\
& +\alpha_{10} \eta \zeta^{2}+\alpha_{11} \eta^{2} \zeta+\alpha_{12} \xi^{2} \zeta+\alpha_{13} \xi \zeta^{2}+\alpha_{14} \xi \eta \zeta \\
& v(\xi, \eta, \zeta)=\beta_{0}+\beta_{1} \xi+\beta_{2} \eta+\beta_{3} \zeta+\beta_{4} \eta \zeta+\beta_{5} \xi \zeta+\beta_{6} \xi \eta+\beta_{7} \xi^{2}+\beta_{8} \eta^{2}+\beta_{9} \zeta^{2} \\
& +\beta_{10} \eta \zeta^{2}+\beta_{11} \eta^{2} \zeta+\beta_{12} \xi^{2} \zeta+\beta_{13} \xi \zeta^{2}+\beta_{14} \xi \eta \zeta \\
& w(\xi, \eta, \zeta)=\gamma_{0}+\gamma_{1} \xi+\gamma_{2} \eta+\gamma_{3} \zeta+\gamma_{4} \eta \zeta+\gamma_{5} \xi \zeta+\gamma_{6} \xi \eta+\gamma_{7} \xi^{2}+\gamma_{8} \eta^{2}+\gamma_{9} \zeta^{2} \\
& +\gamma_{10} \eta \zeta^{2}+\gamma_{11} \eta^{2} \zeta+\gamma_{12} \xi^{2} \zeta+\gamma_{13} \xi \zeta^{2}+\gamma_{14} \xi \eta \zeta
\end{aligned}
$$

Na forma matricial, estas equações são dadas por:

$$
\underset{\sim}{u}=\varphi \cdot \underset{\sim}{\alpha}
$$

sendo:

$$
\begin{gathered}
\underset{\sim}{u}=\left\{\begin{array}{lll}
u & v & w
\end{array}\right\} \\
\varphi=\left[\begin{array}{ccc}
\varphi_{I} & 0 & 0 \\
0 & \varphi_{I} & 0 \\
0 & 0 & \varphi_{I}
\end{array}\right]
\end{gathered}
$$




$$
\begin{aligned}
& \varphi_{I}=\left[\begin{array}{llllllllllllllll}
1 & \xi & \eta & \zeta & \eta & \xi \zeta & \xi \eta & \xi^{2} & \eta^{2} & \zeta^{2} & \eta \zeta^{2} & \eta^{2} \zeta & \xi^{2} \zeta & \xi \zeta^{2} & \xi \eta \zeta
\end{array}\right] \\
& \alpha^{T}=\left\{\begin{array}{lllllllllllllll}
\alpha_{0} & \alpha_{1} & \cdots & \alpha_{13} & \alpha_{14} & \beta_{0} & \beta_{1} & \cdots & \beta_{13} & \beta_{14} & \gamma_{0} & \gamma_{1} & \cdots & \gamma_{13} & \gamma_{14}
\end{array}\right\}
\end{aligned}
$$

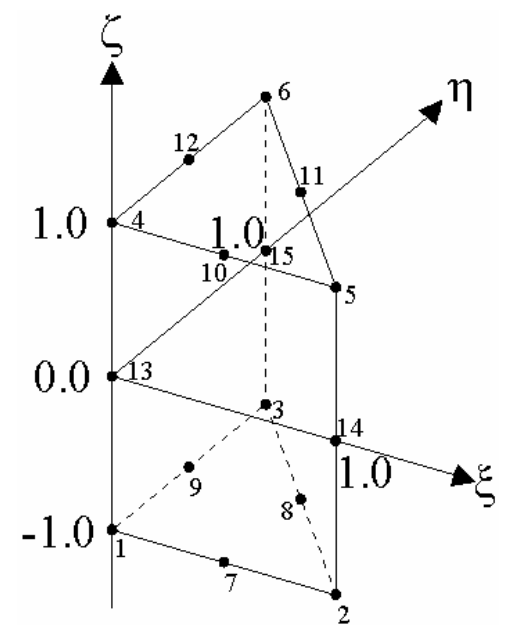

Figura 2 - Valores para as coordenadas nodais - W15. Fonte: Pedreiro (2011)

Particularizando-se para os valores das coordenadas em cada nó na matriz $\underset{\sim}{\varphi}$, de acordo com a Figura 2, tem-se para o Nó $1(\xi=0 ; \eta=0 ; \zeta=-1)$, portanto :

$$
\varphi_{I}=\left[\begin{array}{lllllllllllllll}
1 & 0 & 0 & -1 & 0 & 0 & 0 & 0 & 0 & 1 & 0 & 0 & 0 & 0 & 0
\end{array}\right]
$$

De maneira análoga os vetores $\varphi_{\sim}$ podem ser montados para todos os nós do elemento, conforme demonstrado em PEDREIRO (2011) [4].

Considerando-se conjuntamente os quinze nós do elemento, obtém-se a matriz de coordenadas nodais do elemento apresentada pela equação (13), sendo:

$$
\underset{\sim}{d}=\underset{\sim}{A} \cdot \underset{\sim}{\alpha}
$$

onde $\underset{\sim}{d}$ é dada por:

$$
\underset{\sim}{d^{T}}=\left[\begin{array}{lllllllllll}
u_{1} & v_{1} & w_{1} & \ldots & u_{i+1} & v_{i+1} & w_{i+1} & \ldots & u_{15} & v_{15} & w_{15}
\end{array}\right]
$$

sendo $\underset{\sim}{A}$ :

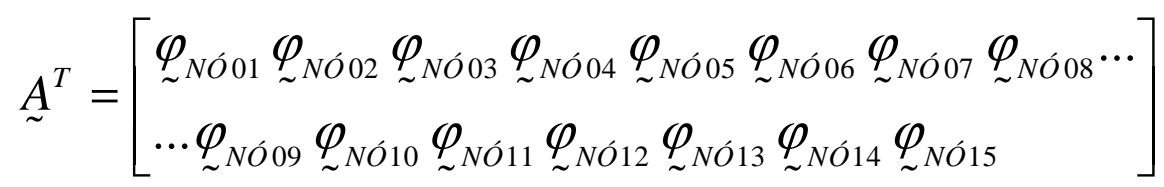

Da equação (13) obtém-se a matriz $\underset{\sim}{\alpha}$, em função da matriz dos deslocamentos $\underset{\sim}{d}$, como mostra a equação (16). 


$$
\underset{\sim}{\alpha}=\underset{\sim}{A}{ }^{-1} \cdot \underset{\sim}{d}
$$

Generalizando-se os deslocamentos em função das coordenadas da estrutura, os deslocamentos $\underset{\sim}{u}$ do elemento finito podem ser expressos em função dos deslocamentos nodais $\underset{\sim}{d}$, por meio da utilização de funções de forma apropriadas, conforme relação definida pela equação (17).

$$
\underset{\sim}{u}=\underset{\sim}{\phi} \cdot \underset{\sim}{d}
$$

Substituindo-se a equação (16) na (7) e comparando-se com a equação (17) obtém-se a matriz das funções de forma $\phi$ para o elemento finito W15 com quinze nós, que é dada pela equação (18).

$$
\underset{\sim}{\phi}=\underset{\sim}{\varphi} \cdot \underset{\sim}{A}
$$

Explicitamente, a matriz $\underset{\sim}{\phi}$ é dada pela equação (19).

$$
\underset{\sim}{\phi}=\left[\begin{array}{ccccccccccc}
N_{1} & 0 & 0 & \ldots & N_{i+1} & 0 & 0 & \cdots & N_{15} & 0 & 0 \\
0 & N_{1} & 0 & \ldots & 0 & N_{i+1} & 0 & \ldots & 0 & N_{15} & 0 \\
0 & 0 & N_{1} & \cdots & 0 & 0 & N_{i+1} & \cdots & 0 & 0 & N_{15}
\end{array}\right]
$$

As funções de forma $\left(\mathrm{N}_{\mathrm{i}}\right)$ são apresentadas em um sistema de coordenadas naturais dadas pelas equações de (20) a (34).

$$
\begin{gathered}
N_{1}=-\frac{(1-\xi-\eta)(1-\zeta)(2 \xi+2 \eta+\zeta)}{2} \\
N_{2}=\frac{\xi(1-\zeta)(2 \xi-\zeta-2)}{2} \\
N_{3}=\frac{\eta(1-\zeta)(2 \eta-\zeta-2)}{2} \\
N_{4}=-\frac{(1-\xi-\eta)(1+\zeta)(2 \xi+2 \eta-\zeta)}{2} \\
N_{5}=\frac{\xi(1+\zeta)(2 \xi+\zeta-2)}{2}
\end{gathered}
$$




$$
\begin{gathered}
N_{6}=\frac{\eta(1+\zeta)(2 \eta+\zeta-2)}{2} \\
N_{7}=2 \xi(1-\xi-\eta)(1-\zeta) \\
N_{8}=2 \xi \eta(1-\zeta) \\
N_{9}=2 \eta(1-\xi-\eta)(1-\zeta) \\
N_{10}=2 \xi(1-\xi-\eta)(1+\zeta) \\
N_{11}=2 \xi \eta(1+\zeta) \\
N_{12}=2 \eta(1-\xi-\eta)(1+\zeta) \\
N_{15}=\eta\left(1-\zeta^{2}\right) \\
N_{13}=\xi(1-\xi-\eta)\left(1-\zeta^{2}\right)
\end{gathered}
$$

A matriz de rigidez de um elemento finito qualquer pode ser deduzida pela equação (35):

$$
\underset{\sim}{k_{S}}=\int_{V_{e}}\left(B_{\sim}^{T} \underset{\sim}{E} \underset{\sim}{B}\right) d V_{e}
$$

onde para elementos sólidos tridimensionais com comportamento elástico linear tem-se: 


$$
\underset{\sim}{E}=\frac{E}{(1+v)(1-2 v)} \cdot\left[\begin{array}{cccccc}
1-v & v & v & 0 & 0 & 0 \\
v & 1-v & v & 0 & 0 & 0 \\
v & v & 1-v & 0 & 0 & 0 \\
0 & 0 & 0 & \frac{(1-2 v)}{2} & 0 & 0 \\
0 & 0 & 0 & 0 & \frac{(1-2 v)}{2} & 0 \\
0 & 0 & 0 & 0 & 0 & \frac{(1-2 v)}{2}
\end{array}\right]
$$

Como a dedução do elemento é feita com a utilização de coordenadas naturais, é necessário mudar o domínio e os limites de integração. Isso é feito através da matriz Jacobiana $\underset{\sim}{J}$ de transformação de coordenadas, que relaciona um elemento infinitesimal do domínio real a um elemento infinitesimal no domínio de coordenadas naturais. A matriz dada pela equação (37) num mapeamento isoparamétrico é chamada de matriz Jacobiana e seu determinante é chamado de Jacobiano, conforme visto em [3].

$$
J=\left[\begin{array}{lll}
\frac{\partial N^{e}}{\partial \xi} x^{e} & \frac{\partial N^{e}}{\partial \eta} x^{e} & \frac{\partial N^{e}}{\partial \zeta} x^{e} \\
\frac{\partial N^{e}}{\partial \xi} y^{e} & \frac{\partial N^{e}}{\partial \eta} y^{e} & \frac{\partial N^{e}}{\partial \zeta} y^{e} \\
\frac{\partial N^{e}}{\partial \xi} z^{e} & \frac{\partial N^{e}}{\partial \eta} z^{e} & \frac{\partial N^{e}}{\partial \zeta} z^{e}
\end{array}\right]
$$

Assim a integral para obtenção da matriz de rigidez explícita do elemento apresentado para o domínio de coordenadas naturais fica definida por:

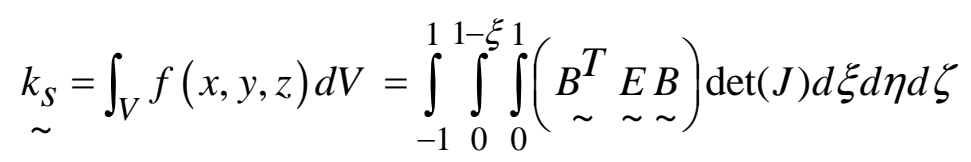

sendo:

$$
\underset{\sim}{B}=\underset{\sim}{L} \cdot \phi
$$

onde $\phi$ é a matriz explicitada em (19) e $L$ é a matriz de operadores de derivação dada pela equaçã̃o (40). 


$$
\stackrel{\sim}{T}^{T}=\left[\begin{array}{cccccc}
\partial / \partial x & 0 & 0 & \partial / \partial y & \partial / \partial z & 0 \\
0 & \partial / \partial y & 0 & \partial / \partial x & 0 & \partial / \partial z \\
0 & 0 & \partial / \partial z & 0 & \partial / \partial x & \partial / \partial y
\end{array}\right]
$$

Fazendo-se as devidas substituições em (39), resulta a equação (41).

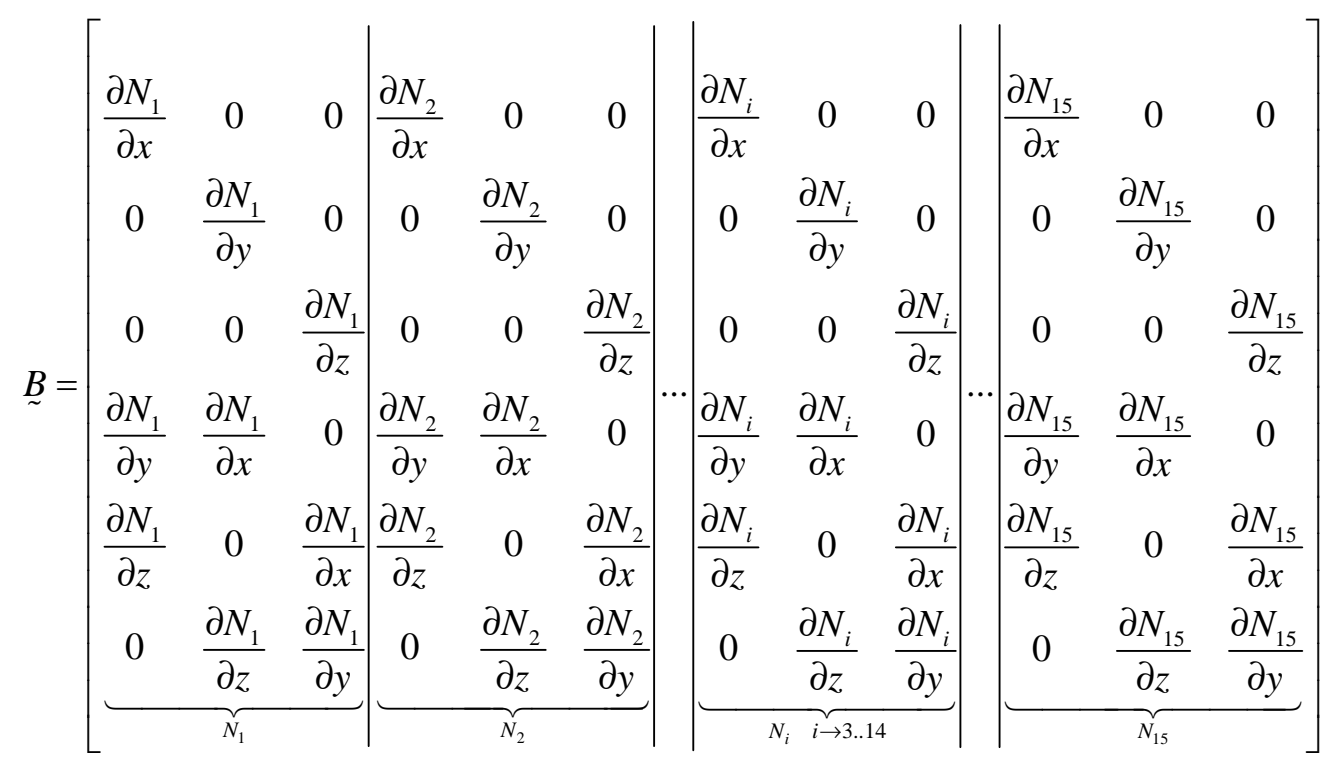

Como as funções de forma do elemento dadas em termos das coordenadas $\xi, \eta$ e $\zeta$, o problema na montagem da matriz $\underset{\sim}{B}$ é que esta contém derivadas das funções de forma com as respectivas coordenadas $\mathrm{x}, \mathrm{y}$ e z. Para obter as derivadas com relação à $\mathrm{x}, \mathrm{y}$ e z na linhagem da matriz, a regra da cadeia da diferenciação parcial deve ser utilizada, sendo expressa na forma matricial pela equação (42):

$$
\left[\begin{array}{l}
\frac{\partial N_{i}}{\partial \xi} \\
\frac{\partial N_{i}}{\partial \eta} \\
\frac{\partial N_{i}}{\partial \zeta}
\end{array}\right]=\left[\begin{array}{l}
\frac{\partial N_{i}}{\partial x} \cdot \frac{\partial x}{\partial \xi}+\frac{\partial N_{i}}{\partial y} \cdot \frac{\partial y}{\partial \xi}+\frac{\partial N_{i}}{\partial z} \cdot \frac{\partial z}{\partial \xi} \\
\frac{\partial N_{i}}{\partial x} \cdot \frac{\partial x}{\partial \eta}+\frac{\partial N_{i}}{\partial y} \cdot \frac{\partial y}{\partial \eta}+\frac{\partial N_{i}}{\partial z} \cdot \frac{\partial z}{\partial \eta} \\
\frac{\partial N_{i}}{\partial x} \cdot \frac{\partial x}{\partial \zeta}+\frac{\partial N_{i}}{\partial y} \cdot \frac{\partial y}{\partial \zeta}+\frac{\partial N_{i}}{\partial z} \cdot \frac{\partial z}{\partial \zeta}
\end{array}\right]=\left[\begin{array}{lll}
\frac{\partial x}{\partial \xi} & \frac{\partial y}{\partial \xi} & \frac{\partial z}{\partial \xi} \\
\frac{\partial x}{\partial \eta} & \frac{\partial y}{\partial \eta} & \frac{\partial z}{\partial \eta} \\
\frac{\partial x}{\partial \zeta} & \frac{\partial y}{\partial \zeta} & \frac{\partial z}{\partial \zeta}
\end{array}\right]\left[\begin{array}{c}
\frac{\partial N_{i}}{\partial x} \\
\frac{\partial N_{i}}{\partial y} \\
\frac{\partial N_{i}}{\partial z}
\end{array}\right]
$$

resultando em: 


$$
\left[\begin{array}{c}
\frac{\partial N_{i}}{\partial x} \\
\frac{\partial N_{i}}{\partial y} \\
\frac{\partial N_{i}}{\partial z}
\end{array}\right]=\left(J^{T}\right)^{-1}\left[\begin{array}{l}
\frac{\partial N_{i}}{\partial \xi} \\
\frac{\partial N_{i}}{\partial \eta} \\
\frac{\partial N_{i}}{\partial \zeta}
\end{array}\right]
$$

onde $\underset{\sim}{J}$ é a matriz Jacobiana de transformação de coordenadas dada num mapeamento isoparamétrico pela equação (37).

No procedimento para obtenção da matriz Jacobiana foram montadas duas matrizes, uma denominada matriz $\underset{\sim}{D}$ que contém a primeira derivada das funções de forma do elemento, dadas em termos das coordenadas $\xi, \eta$ e $\zeta$, e uma matriz $\underset{\sim}{t}$ com as coordenadas nodais expressas em x, y e z, conforme mostrado pela equação (44).

$$
\underset{\sim}{D}=\left[\begin{array}{lllll}
\frac{\partial N_{1}}{\partial \xi} & \frac{\partial N_{2}}{\partial \xi} & \cdots & \frac{\partial N_{14}}{\partial \xi} & \frac{\partial N_{15}}{\partial \xi} \\
\frac{\partial N_{1}}{\partial \eta} & \frac{\partial N_{2}}{\partial \eta} & \cdots & \frac{\partial N_{14}}{\partial \eta} & \frac{\partial N_{15}}{\partial \eta} \\
\frac{\partial N_{1}}{\partial \zeta} & \frac{\partial N_{2}}{\partial \zeta} & \cdots & \frac{\partial N_{14}}{\partial \zeta} & \frac{\partial N_{15}}{\partial \zeta}
\end{array}\right] \quad \underset{\sim}{t}=\left[\begin{array}{ccc}
x_{1} & y_{1} & z_{1} \\
x_{2} & y_{2} & z_{2} \\
\vdots & \vdots & \vdots \\
x_{14} & y_{14} & z_{14} \\
x_{15} & y_{15} & z_{15}
\end{array}\right]
$$

Com base na geometria do elemento, as posições em x e y para os nós 1 e 4, 2 e 5, 3 e 6, 7 e 10, 8 e 11, 9 e 12 são coincidentes tendo variação somente na direção z, e os nós 13, 14 e 15 podem ser obtidos em função dos vértices extremos aos lados em que eles estão inseridos, sendo que a variação para esses nós se dá somente na direção de z.

Com as considerações feitas e sabendo-se que a variação do sistema de coordenadas no eixo z do elemento é definida de -1 até 1 , sendo que o valor da altura do elemento foi estabelecido como 2c, portanto pode-se redefinir a matriz ${\underset{\sim}{t}}^{T}$ conforme equação (45):

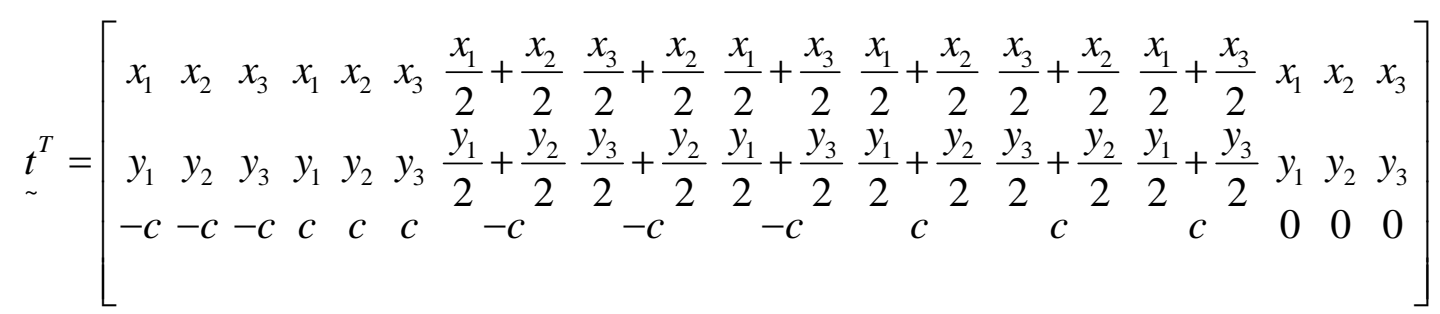

Os termos que compõem a matriz $N$ com a primeira derivada das funções de forma do elemento, dadas em termos das coordenadas $\xi, \eta e \zeta$, as equações 46 a 48 apresentam essas derivadas para o nó 1, sendo que as derivadas para os demais nós podem ser encontradas em PEDREIRO (2011) [4]. 


$$
\begin{gathered}
\frac{\partial N_{1}}{\partial \xi}=2 \xi+2 \eta+\frac{3 \zeta}{2}-2 \zeta \xi-2 \zeta \eta-\frac{\zeta^{2}}{2}-1 \\
\frac{\partial N_{1}}{\partial \eta}=2 \xi+2 \eta+\frac{3 \zeta}{2}-2 \zeta \xi-2 \zeta \eta-\frac{\zeta^{2}}{2}-1 \\
\frac{\partial N_{1}}{\partial \zeta}=\frac{3 \xi}{2}+\frac{3 \eta}{2}+\zeta-\xi^{2}-2 \xi \eta-\zeta \xi-\eta^{2}-\zeta \eta-\frac{1}{2}
\end{gathered}
$$

Realizando-se as multiplicações matriciais e fazendo-se a transposição da matriz obtida, obtém-se a matriz Jacobiana de transformação de coordenadas, dada por:

$$
J=\left[\begin{array}{ccc}
x_{2}-x_{1} & x_{3}-x_{1} & 0 \\
y_{2}-y_{1} & y_{3}-y_{1} & 0 \\
0 & 0 & c
\end{array}\right]
$$

sendo o seu determinante:

$$
\text { Det } J=-c x_{1} y_{3}-c x_{2} y_{1}+c x_{2} y_{3}+c y_{1} x_{3}+c x_{1} y_{2}-c x_{3} y_{2}=2 \text { Area.c }
$$

onde:

Area é a área de a superfície triangular do elemento finito e c é a metade de sua altura, sendo a inversa da equação (49) dada pela equação (51).

$$
J_{\sim}^{-1}=\left[\begin{array}{ccc}
\frac{m_{2}}{2 \text { Area }} & \frac{m_{3}}{2 \text { Area }} & 0 \\
\frac{n_{2}}{2 \text { Area }} & \frac{n_{3}}{2 \text { Area }} & 0 \\
0 & 0 & \frac{1}{c}
\end{array}\right]
$$

Realizando-se a multiplicação matricial dada pela equação (43), obtêm-se as derivadas em função de $\mathrm{x}$, y e z que compõem $\underset{\sim}{B}$, sendo apresentadas para $N 1$ conforme equações 52 a 54, sendo que as demais derivadas podem ser encontradas em PEDREIRO (2011) [4].

$$
\frac{\partial N_{1}}{\partial x}=-\frac{\left(-4 \xi-4 \eta-3 \zeta+4 \zeta \xi+4 \zeta \eta+\zeta^{2}+2\right)\left(m_{2}+m_{3}\right)}{4 \text { Area }}
$$




$$
\begin{gathered}
\frac{\partial N_{1}}{\partial y}=-\frac{\left(-4 \xi-4 \eta-3 \zeta+4 \zeta \xi+4 \zeta \eta+\zeta^{2}+2\right)\left(n_{2}+n_{3}\right)}{4 \text { Area }} \\
\frac{\partial N_{1}}{\partial z}=-\frac{\left(-3 \xi-3 \eta-2 \zeta+2 \xi^{2}+4 \xi \eta+2 \zeta \xi+2 \eta^{2}+2 \zeta \eta+1\right)}{2 c}
\end{gathered}
$$

Conhecendo-se os coeficientes da matriz $\underset{\sim}{B}$ é possível obter a matriz de rigidez do elemento finito W15, com base na equação (38).

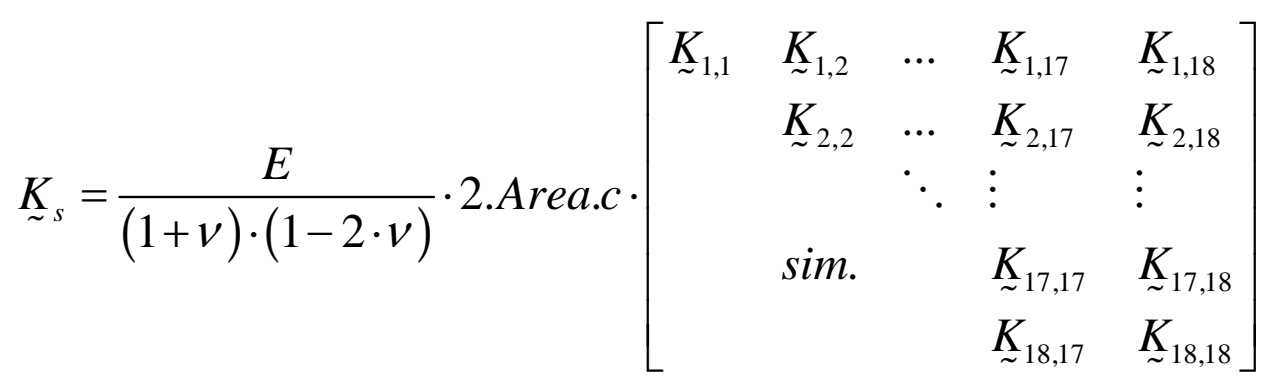

O coeficiente $\underset{\sim 11}{K}$ interno a matriz (55) é apresentado pela equação (56), sendo que os demais termos da matriz de rigidez podem ser encontrados em PEDREIRO (2011) [4], conforme Anexo II.

$$
\underset{\sim 1,1}{K_{1}}=-\frac{1}{48} \cdot \frac{1}{c^{2} \text { Area }^{2}} \cdot\left(\begin{array}{l}
- \text { Area }^{2}+2 \cdot \text { Area }^{2} v-4 c^{2} m_{2}{ }^{2}+4 c^{2} m_{2}{ }^{2} v-8 c^{2} m_{2} m_{3} \\
+8 c^{2} m_{2} m_{3} v-4 c^{2} m_{3}{ }^{2}+4 c^{2} m_{3}{ }^{2} v-2 c^{2} n_{2}{ }^{2}+4 c^{2} n_{2}{ }^{2} v \\
-4 c^{2} n_{2} n_{3}+8 c^{2} n_{2} n_{3} v-2 c^{2} n_{3}{ }^{2}+4 c^{2} n_{3}{ }^{2} v
\end{array}\right)
$$

\section{CARREGAMENTO NODAL EQUIVALENTE - FORÇAS SUPERFICIAIS}

Para expressar a distribuição de uma força uniformemente distribuída aplicada na superfície triangular do elemento finito W15 e obter o vetor de forças nodais equivalentes, define-se:

$$
f_{E}=\int_{S_{e}} N_{\sim}^{T} d S_{e} \underset{\sim}{p_{S_{e}}}
$$

onde

$N_{\sim}$ - são as funções de forma do elemento em termos de x, y e z;

$S_{e}$ - é a superfície do elemento;

$p_{\sim} S_{e}$ - é o vetor de força superficial. 
Considera-se inicialmente uma força uniformemente distribuída na superfície triangular do elemento, conforme Figura 3, apresentando valor da coordenada adimensional na direção $\zeta=1$.

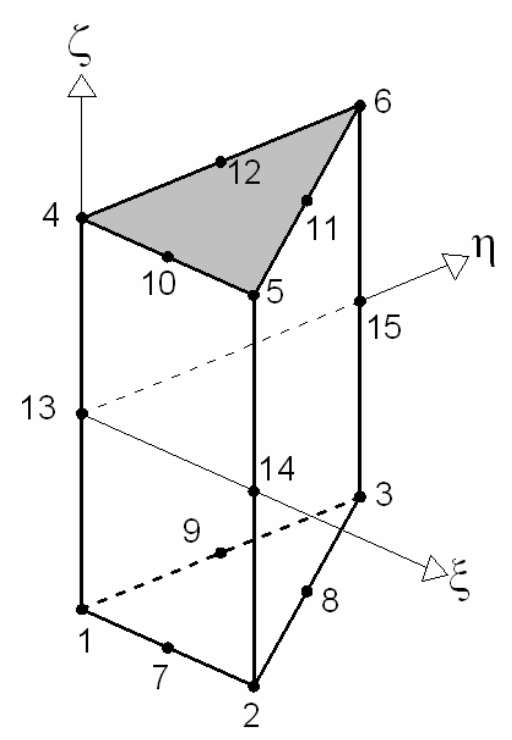

Figura 3 - Superfície a ser carregada

Como as funções de forma são obtidas em função de coordenadas adimensionais, fazse necessário atribuir uma transformação do sistema de coordenadas para cálculo do vetor de forças equivalentes, portanto a expressão para cálculo do vetor de forças fica na forma:

$$
f_{E}=\int_{0}^{1-\xi} \int_{0}^{1} N_{e}^{T} \underset{\sim}{p_{S_{e}}}|a \times b \| d \xi||d \eta|
$$

onde:

$N_{\sim}$ - são as funções de forma do elemento em termos de $\xi, \eta$ e $\zeta$;

a - é a primeira coluna da matriz Jacobiana;

b - é a segunda coluna na matriz Jacobiana.

Considerando-se um carregamento na superfície triangular superior do elemento, conforme mostrado na Figura 4 (a), sabendo-se que para essa superfície $\zeta=1$, a força distribuída na superfície do elemento é dada por:

$$
F=\frac{q_{z}}{\text { Area }}
$$

O vetor das forças nodais equivalentes para a situação apresentada na Figura 4 (a) pode ser dado pela equação (60), ficando distribuído como na Figura 4 (b). 


$$
\underset{\sim}{F} n_{z}^{T}=\left[\begin{array}{llllllllllllllllllllllll}
0 & 0 & 0 & 0 & 0 & -1 / 3 & 0 & 0 & -1 / 3 & 0 & 0 & -1 / 3 & 0 & 0 & 0 & 0 & 0 & 0 & 0 & 0 & 0 & 0 & 0 & 0 \\
0 & 0 & 0 & 0 & 0 & 2 / 3 & 0 & 0 & 2 / 3 & 0 & 0 & 2 / 3 & 0 & 0 & 0 & 0 & 0 & 0 & 0 & 0 & 0 & & &
\end{array}\right] \cdot q_{z}
$$

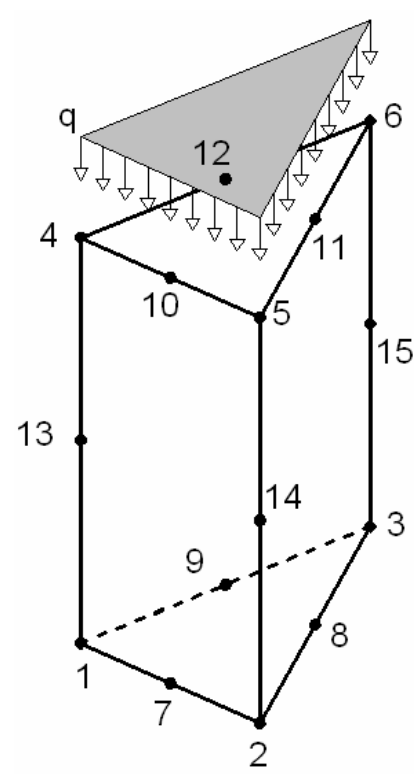

(a)

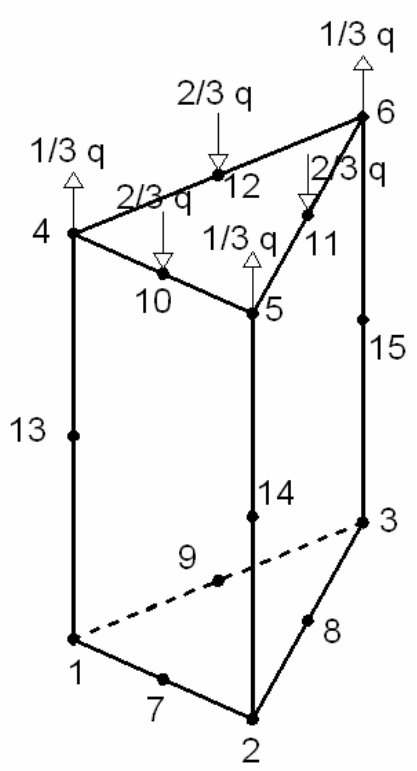

(b)

Figura 4: Carregamento distribuído na superfície (a) e forças nodais equivalentes (b)

\section{ANÁLISE NUMÉRICA COMPARATIVA}

Com o intuito de validar a matriz de rigidez para o elemento finito W15, alguns exemplos são elaborados para efeito comparativo com as equações clássicas da teoria da Resistência dos Materiais. Para todos os exemplos processados são adotados os valores expressos nas equações (61) para as características físicas dos materiais aplicadas aos elementos, ou seja, módulo de elasticidade longitudinal (E) e coeficiente de Poisson (v). Porém para efeito de comparação é necessário considerar o valor do coeficiente de Poisson (v) como sendo nulo, uma vez que a teoria da Resistência dos Materiais também assim o considera.

$$
\mathrm{E}=20000,00 \mathrm{kN} / \mathrm{cm}^{2} \text { e } v=0,00
$$

\subsection{Viga com oito elementos W15 e força axial}

O primeiro exemplo trata de uma viga engastada em uma extremidade e com as forças de superfície distribuídas nos nós da extremidade livre conforme vetor de forças equivalentes, discretizada com o elemento W15. 
Para testar a eficiência do elemento finito W15 com carregamento para esforço normal, foi escolhida aleatoriamente uma força axial de $12 \mathrm{kN}$ aplicada na extremidade livre e distribuída nos nós da estrutura de acordo com a contribuição de cada nó, conforme Figura 4.

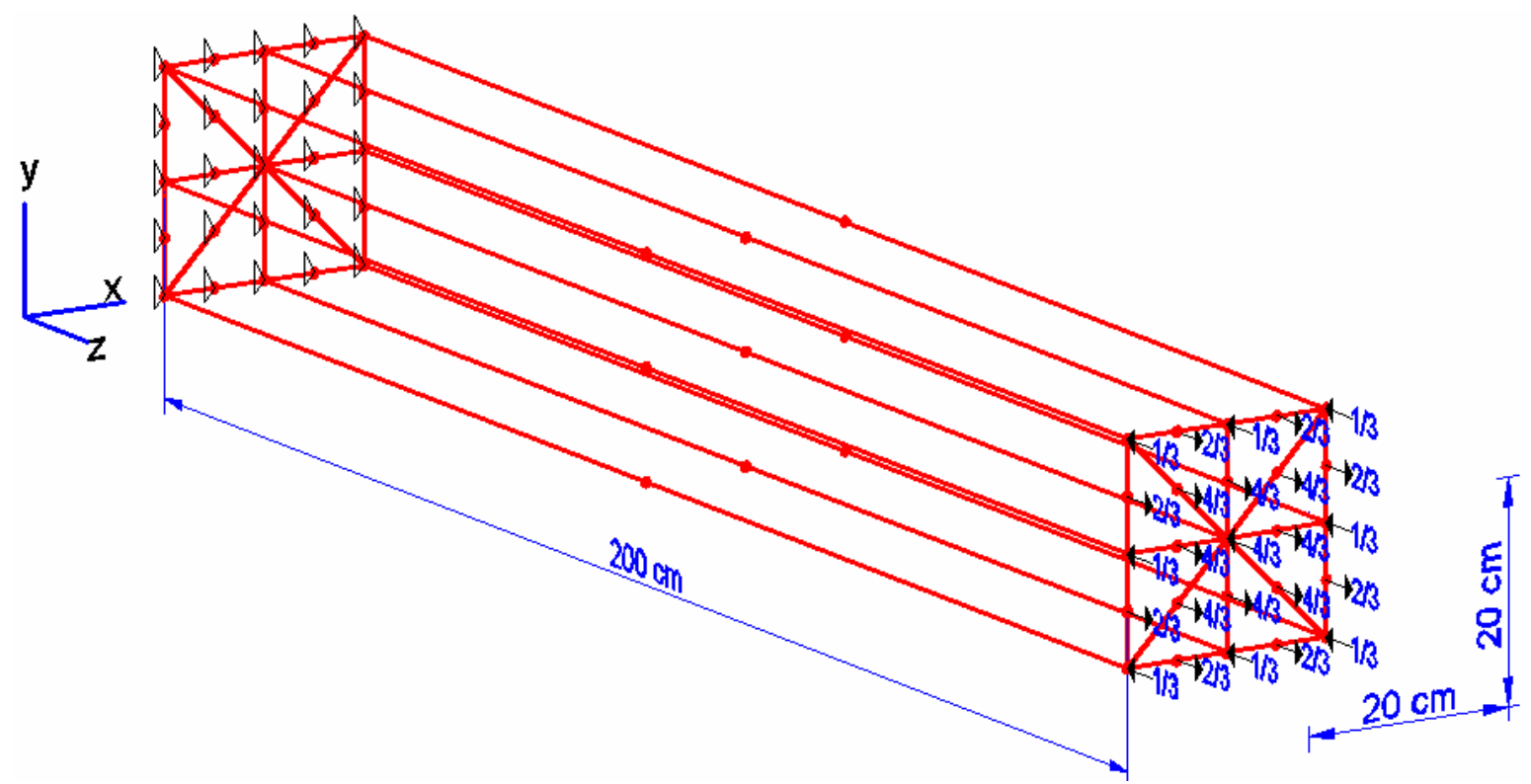

Figura 4 - Discretização da viga com carregamento axial (KN) em z e 8 elementos W15. Fonte: Pedreiro (2011) [4]

Utilizando-se a teoria da Resistência dos Materiais, o deslocamento da extremidade livre de uma barra engastada, submetida a um esforço axial é calculado conforme a equação (62):

$$
\Delta l=\frac{N l}{E A}=\frac{12.200}{20000.400}=0,0003 \mathrm{~cm}
$$

onde:

$\mathrm{N}$ - Esforço normal atuante na barra;

l - Comprimento da barra;

E - Módulo de elasticidade do material;

A - Área da seção transversal da barra.

A Figura 5 mostra o resultado final dos deslocamentos $(\mathrm{cm})$, na direção $\mathrm{z}$ da extremidade livre, obtido pelo código computacional após a execução do exemplo. 


\begin{tabular}{|c|c|c|c|c|}
\hline Deslocame & & & & 미 $x$ \\
\hline Nó & $x$ & $Y$ & $z$ & $\Delta$ \\
\hline 36 & 0 & 0 & 0,00015 & \\
\hline 37 & 0 & 0 & 0 & \\
\hline 38 & 0 & 0 & 0,0003 & \\
\hline 39 & 0 & 0 & 0 & \\
\hline 40 & 0 & 0 & 0 & \\
\hline 41 & 0 & 0 & 0,0003 & \\
\hline 42 & 0 & 0 & 0,0003 & \\
\hline 43 & 0 & 0 & 0,00015 & \\
\hline 44 & 0 & 0 & 0 & \\
\hline 45 & 0 & 0 & 0,0003 & \\
\hline 46 & 0 & 0 & 0 & \\
\hline 47 & 0 & 0 & 0 & \\
\hline 48 & 0 & 0 & 0,0003 & \\
\hline 49 & 0 & 0 & 0,0003 & \\
\hline 50 & 0 & 0 & 0,00015 & \\
\hline 51 & 0 & 0 & 0 & \\
\hline 52 & 0 & 0 & 0,0003 & \\
\hline 53 & 0 & 0 & 0 & \\
\hline 54 & 0 & 0 & 0 & \\
\hline 55 & 0 & 0 & 0,0003 & \\
\hline 56 & 0 & 0 & 0,0003 & \\
\hline 57 & 0 & 0 & 0,00015 & \\
\hline 58 & 0 & 0 & 0 & \\
\hline 59 & 0 & 0 & 0,0003 & $\nabla$ \\
\hline
\end{tabular}

Figura 5 - Resultados dos deslocamentos nodais (cm) para discretização com 8 elementos W15 e carregamento axial. Fonte: Pedreiro (2011) [4]

Pode-se observar que os deslocamentos dos nós da extremidade livre, na direção z, após a aplicação do carregamento, são iguais a $0,003 \mathrm{~cm}$, que corresponde exatamente ao resultado apresentado na equação (62).

\subsection{Viga com oito elementos W15 e força perpendicular ao eixo}

Neste segundo exemplo foi testada a eficiência do elemento W15 submetido à flexão, com carregamento perpendicular ao eixo com magnitude de $12 \mathrm{kN}$, escolhido aleatoriamente, distribuído de acordo com a contribuição de cada nó da estrutura, aplicado na extremidade livre, conforme Figura 6. 


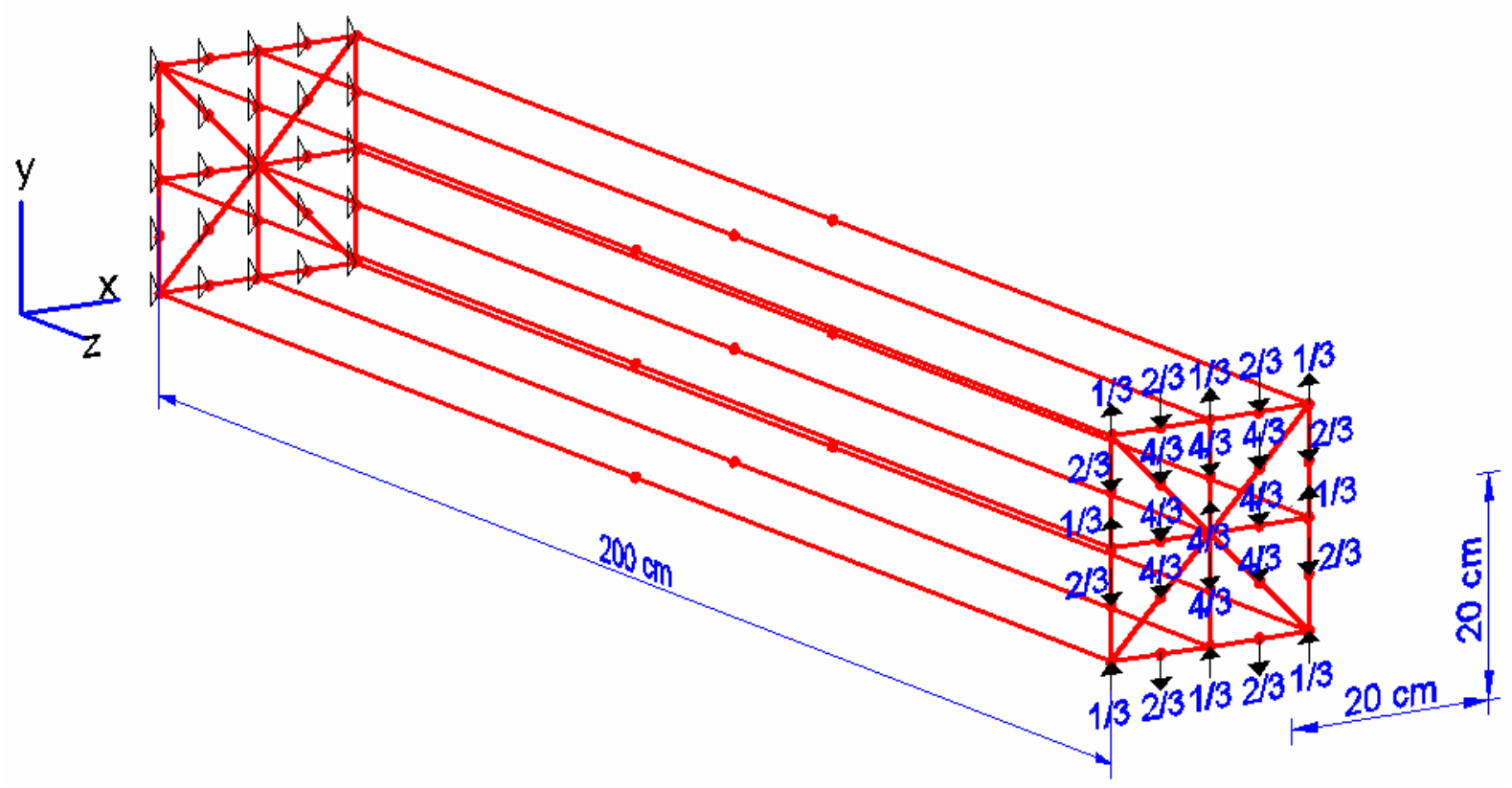

Figura 6 - Discretização da viga com carregamento perpendicular ao eixo (KN) e 8 elementos W15. Fonte: Pedreiro (2011) [4]

De acordo com a teoria da Resistência dos Materiais, o deslocamento máximo de uma viga engastada, submetida a carregamento perpendicular ao seu eixo e aplicado na extremidade livre, é calculado conforme a equação (63):

$$
v=\frac{p \cdot l^{3}}{3 E I}=\frac{-12 \cdot 200^{3}}{3 \cdot 20000 \cdot 13333,33}=-0,12 \mathrm{~cm}
$$

onde:

p - Esforço perpendicular ao eixo atuante na barra;

l - Comprimento da barra;

E - Módulo de elasticidade do material;

I - Momento de Inércia da seção transversal.

A Figura 7 mostra o resultado final dos deslocamentos (cm), na direção y, dos nós na extremidade livre obtidos pelo código computacional após a execução do exemplo. 


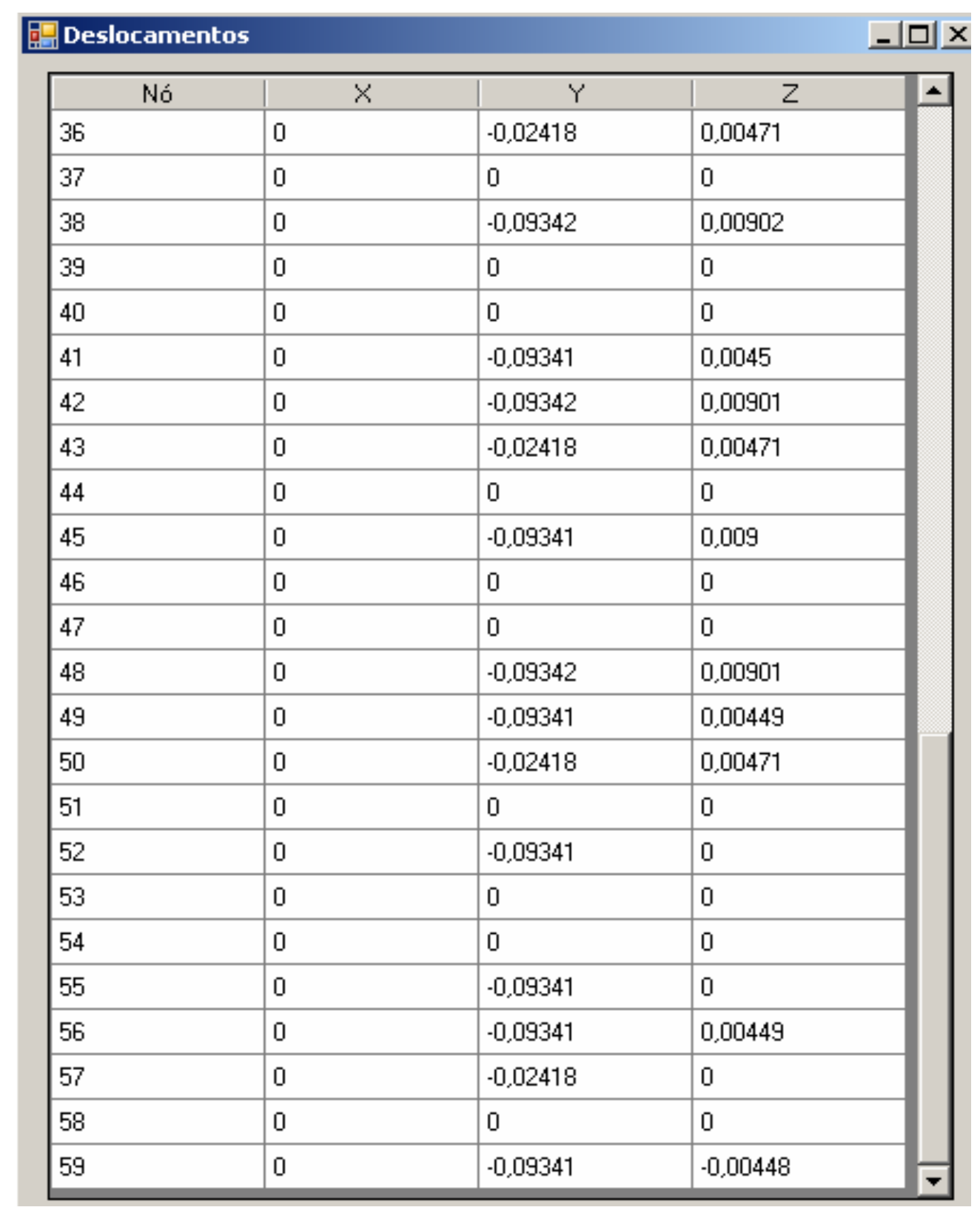

Figura 7 - Resultados dos deslocamentos nodais (cm) para discretização com 8 elementos W15 e carregamento (KN) perpendicular ao eixo. Fonte: Pedreiro (2011) [4]

Pode-se observar que os deslocamentos dos nós da extremidade livre, na direção y, após a aplicação do carregamento, são aproximadamente iguais a -0,09341 cm. Conclui-se que a discretização foi razoavelmente suficiente para o tipo de esforço aplicado.

\subsection{Viga com dezesseis elementos W15 e força perpendicular ao eixo}

Com o intuito de melhorar os resultados, optou-se então por realizar uma discretização maior na direção do eixo longitudinal da estrutura (eixo z). A Figura 8 mostra a viga discretizada com duas camadas, utilizando oito elementos W15 por camada. 


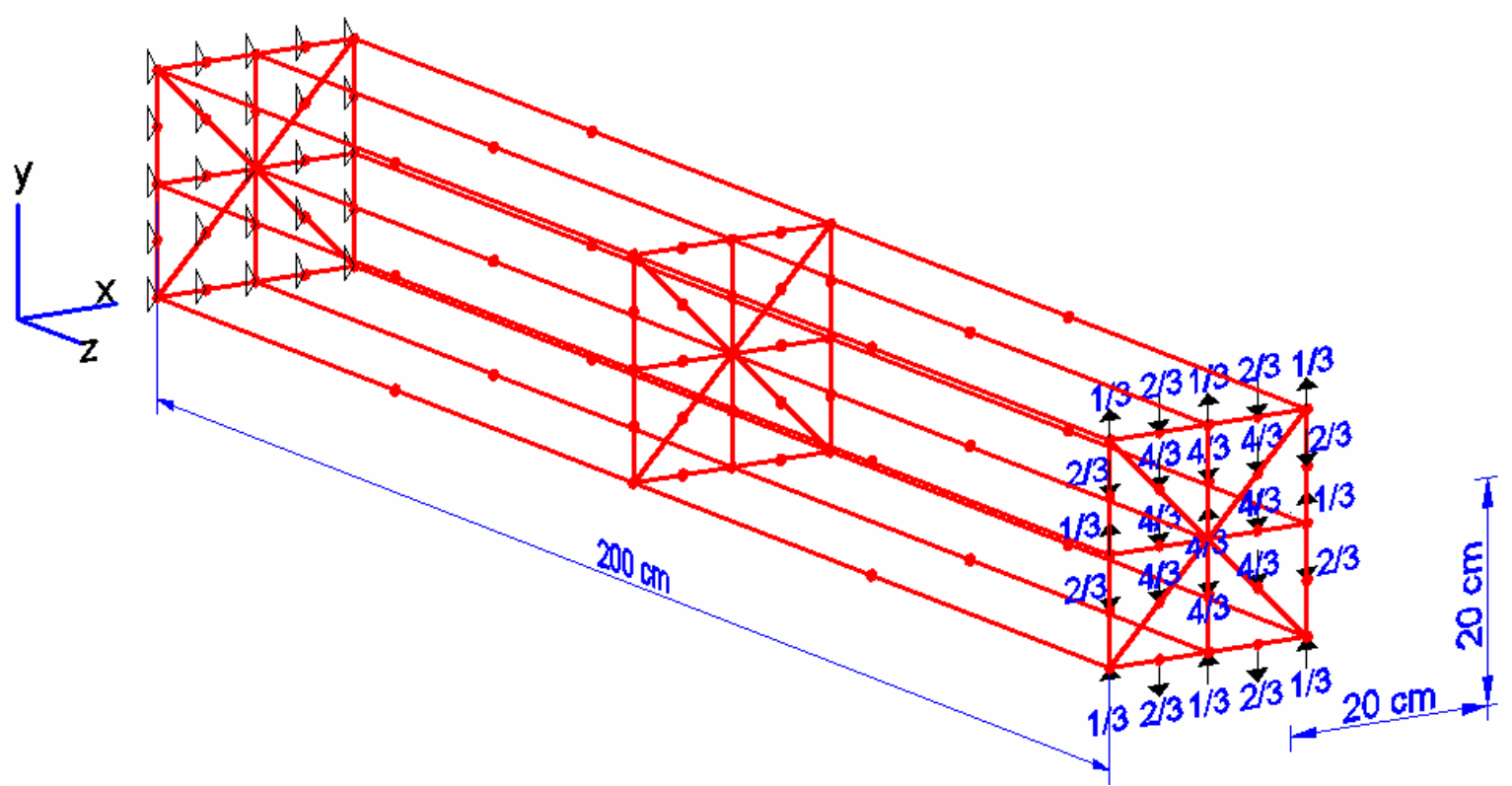

Figura 8 - Discretização da viga com carregamento perpendicular ao eixo (KN) e 16 elementos W15. Fonte: Pedreiro (2011) [4]

A Figura 9 mostra o resultado final dos deslocamentos $(\mathrm{cm})$, na direção y, na extremidade livre obtidos pelo código após a execução do exemplo com 2 camadas de 8 elementos W15.

\begin{tabular}{|c|c|c|c|c|}
\hline Deslocame & & & & 미 \\
\hline Nó & $\bar{x}$ & $\bar{Y}$ & $z$ & $\Delta$ \\
\hline 70 & 0 & $-0,11535$ & $-0,009$ & \\
\hline 71 & 0 & $-0,11535$ & $-0,00448$ & \\
\hline 72 & 0 & $-0,07244$ & $-0,00804$ & \\
\hline 73 & 0 & $-0,11535$ & 0 & \\
\hline 74 & 0 & $-0,11535$ & 0 & \\
\hline 75 & 0 & $-0,11535$ & $-0,00448$ & \\
\hline 76 & 0 & $-0,07244$ & 0 & \\
\hline 77 & 0 & $-0,11535$ & 0,009 & \\
\hline 78 & 0 & $-0,11535$ & 0,00449 & \\
\hline 79 & 0 & $-0,11535$ & 0,00449 & \\
\hline 80 & 0 & $-0,07244$ & 0,00805 & \\
\hline 81 & 0 & $-0,11535$ & 0,00902 & \\
\hline 82 & 0 & $-0,11535$ & 0,0045 & \\
\hline 83 & 0 & $-0,11535$ & 0,00901 & \\
\hline 84 & 0 & $-0,07244$ & 0,00804 & \\
\hline 85 & 0 & $-0,11535$ & 0,009 & \\
\hline 86 & 0 & $-0,11535$ & 0,00901 & \\
\hline 87 & 0 & $-0,11535$ & 0,00449 & \\
\hline 88 & 0 & $-0,07244$ & 0,00805 & \\
\hline 89 & 0 & $-0,11535$ & 0 & \\
\hline 90 & 0 & $-0,11535$ & 0 & \\
\hline 91 & 0 & $-0,11535$ & 0.00449 & \\
\hline 92 & 0 & $-0,07244$ & 0 & \\
\hline 93 & 0 & $-0,11535$ & $-0,00448$ & $\nabla$ \\
\hline
\end{tabular}

Figura 9 - Resultados dos deslocamentos nodais (cm) para discretização com 16 elementos W15 e carregamento (KN) perpendicular ao eixo. Fonte: Pedreiro (2011) [4] 
Pode-se observar que os deslocamentos dos nós da extremidade livre, na direção y, após a aplicação do carregamento, são iguais a $-0,11535 \mathrm{~cm}$. Com essa discretização os resultados mostraram-se muito satisfatórios, uma vez que convergem para a resposta obtida pela equação (63).

\section{CONCLUSÃO}

O trabalho apresentou a dedução da matriz de rigidez explicita do elemento finito W15, bem como a dedução do vetor de forças nodais equivalentes explicito para esforços uniformemente distribuídos e aplicados na superfície triangular. Os exemplos simulados numericamente e submetidos a forças axiais, com modelo discretizado pelo elemento proposto, apresentaram resultados idênticos quando comparados com os deslocamentos obtidos pela teoria clássica de Resistência dos Materiais. Já os exemplos simulados numericamente e submetidos a esforços perpendiculares ao eixo da viga apresentaram resultados muito bons, pois somente com a discretização de um elemento na direção do eixo da viga os valores obtidos foram bem razoáveis. Na continuidade, com a discretização de dois elementos na direção do eixo da viga os valores obtidos foram excelentes, apresentando excelente convergência. Com base no exposto, pode-se validar a matriz de rigidez explicita obtida e o vetor de forças nodais equivalentes com base nas comparações efetuadas com as equações clássicas da teoria de Resistência dos Materiais, podendo-se utilizar o elemento W15 explicito na discretização dos mais diversos modelos. 


\section{REFERÊNCIAS}

[1] Albertini, M. M. Análise do comportamento experimental e numérico de prismas de alvenaria estrutural utilizando o elemento finito prismático regular parabólico. 2009. 214f. Dissertação (Mestrado em Engenharia Civil) - Faculdade de Engenharia, Universidade Estadual Paulista “Júlio de Mesquita”, Ilha Solteira, 2009.

[2] Bathe, K. Finite element procedures in engineering analysis, Prentice-Hall, Inc., 1982. New Jersey. United States of America.

[3] Cook, R.D. Finite element modeling for stress analysis. John Wiley \& Sons, INC, 1995. Unites States of America.

[4] Pedreiro, M. R. M. Análise do comportamento experimental e numérico de prismas de alvenaria estrutural submetidos a ações verticais utilizando elementos finitos volumétricos. 2011. 209f. Dissertação (Mestrado em Engenharia Civil) - Faculdade de Engenharia, Universidade Estadual Paulista “Júlio de Mesquita”, Ilha Solteira, 2011.

[5] Rodrigues, R. O. Análise Dinâmica Bidimensional Não-Linear Física e Geométrica de Treliças de Aço e Pórticos de Concreto Armado. 1997. 298f. Tese (Doutorado em Engenharia de Estruturas) - Escola de Engenharia de São Carlos - Universidade de São Paulo, São Carlos, 1997.

[6] Zienkiewicz, O. C.; Taylor, R. L. The finite element method. $5^{\mathrm{a}}$ ed. Oxford: ButterworthHeinemann, 2000. 3v. 